\title{
Radioimmunotherapy: future prospects from the perspective of brachytherapy
}

\author{
Maximilian Fleischmann, MD', Markus Glatzer, MD², Prof. Claus Rödel, MD',3,4, Nikolaos Tselis, MD, PhD' \\ 'Department of Radiation Oncology, University Hospital Johann Wolfgang Goethe University, Frankfurt, Germany, 2Department of \\ Radiation Oncology, Kantonsspital St. Gallen, St. Gallen, Switzerland, ${ }^{3}$ German Cancer Research Center (DKFZ), Heidelberg, German Cancer \\ Consortium (DKTK), Partner Site Frankfurt am Main, Frankfurt, Germany, "Frankfurt Cancer Institute, Frankfurt, Germany
}

\begin{abstract}
In combination with radiotherapy, immunotherapy is becoming an increasingly used strategy in treating advanced, recurrent, or metastatic cancer. The evident impact of radiotherapy on local and systemic immune response is an indication of the synergistic effect of these two modalities. There is a strong rationale to combine radiotherapy and immunotherapy to enhance response rates and overcome resistances. Therefore, the combination of radio- and immunotherapy holds a variety of opportunities as well as challenges in treating primary cancer and is progressively tested in curative settings. Brachytherapy is also known as internal radiation therapy and only offers a local therapy option at first glance: due to tumor-specific antigens, released by a high local radiation dose, a systemic immune response could be plausible and eminent. Accordingly, brachytherapy could be an underestimated partner with immuno-therapeutic approaches in both curative and palliative settings, to generate local and systemic response. In this review, we summarized the potential benefit of a potential combination of brachytherapy and immuno-therapeutic approaches vs. the background of limited data.

J Contemp Brachytherapy 2021; 13, 4: 458-467 DOI: https://doi.org/10.5114/jcb.2021.108601
\end{abstract}

Key words: brachytherapy, immunotherapy, radioimmunotherapy, abscopal effect.

\section{Purpose}

Immunotherapy rapidly became an integral part of multimodal treatment concepts in modern cancer therapy and its implementation is constantly evolving [1]. Immunotherapy can be defined as treatment approach that influences and enhances anti-tumor immune response. Immune checkpoint inhibitors that intervene in inhibitory signaling pathways on immune cells [cytotoxic T-lymphocyte-associated protein 4 (CTLA-4) and programmed cell death 1/ligand (PD-1/PD-L1)], have been approved and are frequently used to date [2]. New concepts for combinatorial therapies using immune checkpoint inhibitors are emerging, especially approaches combining checkpoint inhibitors and radiotherapy. In this context, PACIFIC trial, in which the immune checkpoint inhibitor durvalumab was used as an effective and well-tolerated consolidation therapy following chemoradiotherapy in patients with primarily unresectable non-small-cell lung carcinoma (NSCLC), showed promising oncological outcomes [3]. Currently, immune checkpoint inhibitors are routinely used in patients with melanoma, urothelial carcinoma, and head and neck tumors [4-7], while a large number of studies are examining application of checkpoint inhibitors in other entities. Besides immune checkpoint inhibitors and CAR-T cells, which have already been successfully established, there are several other immuno-therapeutic approaches, including OX-40, CDX-301, or Toll-like receptor (TLR) agonists as well as therapeutic vaccines, which differ considerably in their mechanism of action and biological behavior; they have not yet reached regular clinical use and are only available in experimental studies [8].

Despite promising pre-clinical results, a durable tumor response under immunotherapy is not observed in all patients and can rarely be predicted [9]. Nevertheless, a number of biomarkers exist to predict the therapeutic response to immune checkpoint inhibitors. Besides the exact stratification of suitable patients, a combination with radiotherapy can be a potential approach to improve the efficacy of immunotherapies, and lead to synergistic effects of both modalities. This synergism is based on immunomodulatory effects of radiotherapy [10]. The majority of pre-clinical data investigating the synergistic effect of immunotherapy in combination with radiotherapy have used external beam radiotherapy (EBRT). As radiation-induced immune response seems to be influenced by timing, dose, target volume, and fractionation of radiotherapy, brachytherapy may be advantageous over 
EBRT in this context [11-13]. Brachytherapy enables highly conformal dose distribution by providing high, but also heterogeneous intra-tumoral radiation dose. This heterogeneity depends on the distance of the source from the target volume. Then, different dose levels lead to various effects; while lower doses lead to a temporary depletion of tumor-infiltrating lymphocytes (TILS), cytokine release, and immune cell infiltration, high doses result in direct or indirect cell death and finally to the release of tumor antigens [14]. Therefore, the combination of brachy- and immunotherapy seems to offer an additional interesting possibility to improve treatment of patients in both definitive and palliative settings. In this article, we focused on these possibilities and reviewed the current status and future perspectives of combining brachy- and immunotherapy.

\section{Current status and approaches in targeting immune response}

\section{Immuno-therapeutic agents}

Immune checkpoint inhibitors are blocking crucial inhibitory signaling pathways, which are controlling the activation of T cells, and thereby promote anti-tumor effects. Since the first approval of pembrolizumab in 2014, five additional monoclonal antibodies targeting PD-1 or its ligand PD-L1 have been permitted by the Food and Drug Administration/European Medicines Agency (FDA/ EMA). Meanwhile, the number of active clinical trials has increased rapidly. The majority of this expanding landscape of clinical trials includes combinations of antiPD-1/PD-L1 agents with other cancer therapies. In 2019, there was a total of 2,975 active trials, of which 2,251 investigated a combination of anti-PD-1/PD-L1 with other modalities. Most of these studies examined a combination of anti-PD-1/PD-L1 with anti-CTLA4 agents, followed by a combination with chemotherapy and anti-angiogenic agents targeting the vascular endothelial growth factor receptor (VEGFR) axis [15].

In addition to immune checkpoint inhibitors, there are numerous other approaches to influence and enhance antitumor immune response. For example, T cell stimulating immuno-therapeutic agents, such as OX-40 agonists, genetically modified T cells (CAR-T cells), and bi-specific antibodies as well as vaccination approaches against tumorspecific or tumor-associated antigens. Furthermore, the tumor micro-environment can be influenced by immunostimulating cytokines, transforming growth factor $\beta$ (TGF- $\beta$ ) blockade, or TLR agonists [16].

\section{Immuno-genetic effects of radiotherapy/ brachytherapy}

For local tumor irradiation, immuno-stimulatory and immuno-suppressive effects have been reported [10].

Pro-immunogenic properties of radiotherapy are described by aspects, such as the abscopal effect (tumor regression in non-irradiated lesions) or the in situ vaccination (enhancing immunogenicity of an immuno-suppressive tumor micro-environment), including both local and systemic immune responses $[17,18]$. According to systemic immune response, cell death of tumor cells triggered by irradiation has immuno-genic properties [19]. Tumor-specific antigens and danger signals, with inflammatory cytokines and damage-associated molecular patterns are released, causing activation of innate and subsequently, the adaptive immune system. MHC-1 molecules in the tumor micro-environment are up-regulated, improving the "visibility" of tumor cells for the immune system [20]. In addition, radiotherapy can induce a pro-angiogenic shift [21]. Changes in the endothelium of the tumor blood vessels caused by irradiation, lead to an adhesion of circulating immune cells and facilitate the immigration of effector cells into tumor tissue [22]. In particular, induction of SDF1 leads to infiltration of myeloid cells and TIE2-expressing monocytes, resulting in increased angiogenesis. These immuno-suppressive and pro-angiogenic tumor-associated macrophages could promote tumor recurrence and diminish therapeutic benefit [23-25]. Especially hypofractionated radiotherapy can alter the extracellular matrix and vascular permeability by promoting reconstitution of the tumor vasculature. As already mentioned, this may improve immune cell infiltration and reduce tumor hypoxia. Apart from that, high-dose radiotherapy, such as stereotactic body radiation therapy (SBRT), is considered to induce severe vascular damage, leading to indirect tumor cell death [26-28]. Effected by these phenotypic changes, $\mathrm{T}$ cell response is further enhanced by the maturation of dendritic cells and antigen-cross-presentation [29]. Even though in vitro experiments and pre- and post-therapeutic patients' samples have shown that the infiltration of anti-tumor immune effector cells significantly increased in irradiated tumors as compared to non-irradiated tumors, irradiation leads to a temporary depletion of radiation-sensitive immune lineages, including CD4- and CD8-positive lymphocytes, which can result in long-term lymphopenia [30]. Moreover, irradiation leads to an upregulation of PD-L1 in the tumor tissue and can stimulate the secretion of various pro-inflammatory cytokines and chemokines, such as TGF- $\beta$, chemokine CC motif ligand 2 (CCL2), colony-stimulating factor 1 (CSF-1), CXC motif chemokine ligand 12 (CXCL12), or insulin-like growth factor 1 (IRF1) [31-33].

In general, higher radiation doses seem to have more immune-stimulating effects, while lower radiation doses $(<2$ Gy) may present with more immuno-suppressive and anti-inflammatory effects [34]. Therefore, brachytherapy may have potential advantages over EBRT in achieving tumor immuno-modulation. While EBRT passes through considerably volumes of healthy tissue to reach targeted tumor volumes, brachytherapy enables a highly conformal dose distribution by providing a high, but also heterogeneous intra-tumoral radiation dose (Figure 1). This heterogeneity depends on the distance of the source from the target volume [14]. Patel et al. assumed that highly conformal dose distribution enables sparing of normal tissue, especially circulating immune components and lymphatic tissue, which minimizes off-target effects. Instead, in the target itself, a dose gradient is created according to linear quadratic formulation depending on the distance to the source. This causes multiple immuno-gen- 


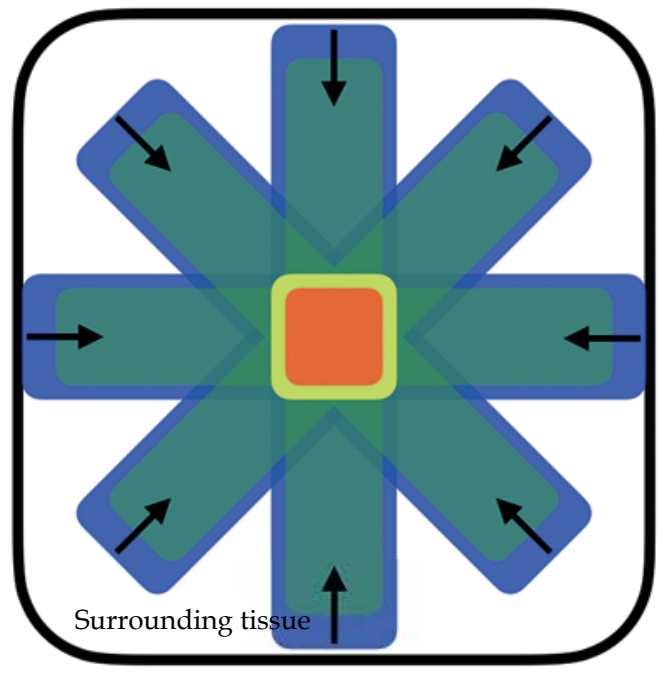

External beam radiotherapy (EBRT)

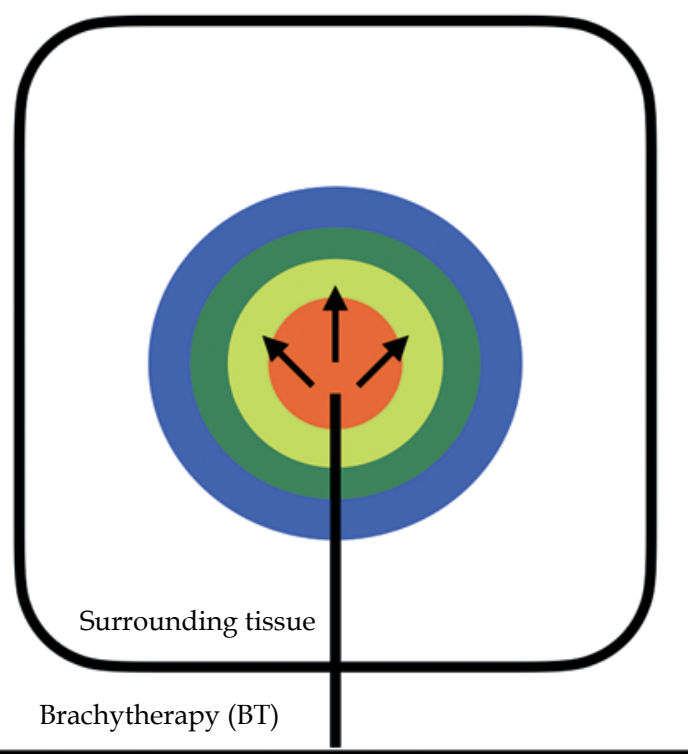

Brachytherapy (BT)

Low-dose region (<2 Gy): temporary depletion of immune cell lines, such as tumor-infiltration lymphocytes (TILs).

Moderate-dose region (2-5 Gy): cytokine release is leading to enhanced immune cell infiltration to tumor micro-environment.

Intermediate-dose region (5-8 Gy): phenotypic changes in immune marker expression and release of cytoplasmic dsDNA.

High-dose region (> $8 \mathrm{~Gy}$ ): tumor cell death and release of tumor-specific antigens.

Fig. 1. Comparative, schematic illustration of dose distribution in external beam radiotherapy (EBRT) vs. brachytherapy (BT). Brachytherapy allows for highly conformal dose distribution and optimal sparing of organs at risk (OARs), including blood vessels, lymphatic tissue, and bone marrow

ic mechanisms, ranging from immuno-genic cell death and the release of tumor-specific antigens in regions of the highest dose, to an increased release of cytokines in the medium-dose area, and finally, to a short-term depletion of suppressive tumor-infiltrating lymphocytes in surrounding tumor tissues [34].

\section{Combination of immunotherapy agents and radiotherapy/brachytherapy}

There is ample evidence for important interplay between radiotherapy and the immune system [35]. Reports on abscopal effects (tumor regression in non-irradiated lesions) in patients with metastatic tumors receiving immune checkpoint inhibitors and undergoing local radiotherapy, are supporting the thesis of immune-modulatory effect of irradiation [36-38].

For example, cytokines are a diverse family of signaling glycoproteins of the immune system, which enable the immune cells to communicate with each other and influence the surrounding micro-environment in autocrine, endocrine, and paracrine manners. Such a communication plays an important role in the regulation and control of immune reactions. In that way, cytokines can influence the activity of immune cells and regulate their maturation and reproduction. In a pre-clinical murine tumor model, intra-tumoral delivery of cytokines seems to enhance the in situ vaccination effect of EBRT. In combination with anti-CTLA4 agents, further synergistic effects could be achieved [39]. Moreover, subcutaneous injections of granulocyte-macrophage colony-stimulating factor (GMCFS) in combination with EBRT (35 Gy in 10 fractions) in $26.8 \%$ of 41 patients with metastatic solid tumors, have generated abscopal tumor response [38]. However, the anti-tumor effect of cytokines in combination with radiotherapy could not fulfill the initially high expectations, especially the increased toxicity has been a problem after systemic administration of these agents [40]. At present, only a few cytokines are used for a limited number of oncologic indications and in a small number of early phase clinical trials, in which interleukin 2 (IL-2), interferon $\alpha$ (IFN- $\alpha$ ), GM-CSF, and tumor necrosis factor $\alpha$ (TNF- $\alpha$ ) are analyzed as a combinative treatment with radiotherapy. Others investigate intra-tumoral administration of cytokines, decreased toxicity, and relevant efficacy.

Irradiation induces an up-regulation of PD-L1 in tumor tissue and the secretion of various pross-inflammatory cytokines and chemokines, such as TGF- $\beta$. These facts are important arguments for combining radiation with immuno-therapeutic agents, including PD-1/PD-L1 blockade and TGF- $\beta$ blockers, as in this way the pro-immunogenic properties of radiation can be exploited while blocking further immunosuppression [41]. A variety of 
ongoing clinical trials are currently investigating the efficacy of SBRT combined with PD-1/PD-L1 blockade in patients with NSCLC, and this combination has shown promising results and good response rates in pre-clinical and clinical studies, especially in metastatic disease stages [42]. In advanced stage non-small cell lung cancer, for example, a recent, single-center unexpected posthoc analysis of KEYNOTE-001 trial [35] demonstrated a benefit in terms of progression-free survival (PFS) and overall survival (OS) in patients with metastatic NSCLC who received pembrolizumab and had previously underwent radiotherapy, compared to those who had not underwent radiotherapy, thereby overcoming the effect of PD-L1 expression in multivariate analysis [43]. Similarly, a single-center cohort study of patients with metastatic lung cancer receiving PD-1 or PD-L1 inhibitors found that there was a trend for longer overall survival in patients with prior (or concurrent) thoracic radiotherapy than patients without radiotherapy [44]. The PEMBRO-RT, a randomized, multi-center phase 2 clinical trial, reported a two-fold increase in the proportion of patients with metastatic NSCLC who achieved an objective response after PD-1 blockade and SBRT of previously non-irradiated NSCLC lesions. There was no increased toxicity compared with PD-1 blockade alone. Despite the augmenting effect of SBRT on PD-1 blockade response, no meaningful clinical benefit was observed. However, in a sub-group of PD-L1-negative patients, a significant benefit was observed [45]. Nevertheless, these data indicate that in oligometastatic settings, combinatorial therapies improve therapeutic response rates and may only cause a low-rate of acute and late toxicities [46]. Toxicities from immunotherapies are enormously diverse, but a recent meta-analysis was able to show that the combination of immune checkpoint inhibitors and radiotherapy has a comparable toxicity to immune checkpoint inhibitors alone. Accordingly, the combination of these two modalities did not show to significantly increase toxicity [47]. However, a sequential administration of multiple therapeutic modalities could be favorable when a tri-modal therapy approach is intended. In particular, a combination of chemoradiotherapy and immunotherapy could have a considerably rate of cumulative toxicities, so that a sequential administration could be advantageous over a simultaneous administration [48].

While most of these studies included EBRT, there is only a small number of trials including brachytherapy, reflecting the predominant role of EBRT [34] (Table 1). Nevertheless, as described above, brachytherapy may have the potential to influence the immune system in a positive manner.

Pre-clinical approaches seem to support this consideration. In a carcinoembryonic antigen-positive (CEA+) mouse model, a CEA-directed vaccine was used in a combination with brachytherapy by implanting iodine- 125 $\left.{ }^{(125} \mathrm{I}\right)$ seed into the primary tumor site. The combination of both modalities led to a significant reduction of metastatic burden in the lungs, while neither vaccination nor brachytherapy alone could cause a similar effect [49]. In another pre-clinical report, Rodriguez-Ruiz et al. combined brachytherapy with anti-PD-1 and CD137 antibodies, inducing a cross-priming in a mouse model. Only the triplet of brachytherapy and anti-PD-1 and CD137 antibodies generated a response in a contralateral and therefore, non-irradiated tumor lesion, suggesting an abscopal regression [50]. In addition, pre-clinical data shows an elevated expression of PD-L1 in a time interval after high-dose brachytherapy, demonstrating immuno-modulatory effect of this treatment modality and suggesting the use of PD-1/PD-L1 checkpoint blockade [51]. In a clinical study, with the objective to assess effectiveness of diffusing alpha emitters radiation therapy (DaRT), a spontaneous regression of untreated distant lesions was observed in a patient with multiple SCC lesions after treatment of a single-lesion with DaRT and with no immuno-therapeutic agent. This observation provides new evidence for an abscopal effect after local treatment with an alpha emitter, and suggests an activation of immune system mediated by radiation [52]. Finally, Suzuki et al. have reported the first abscopal effect after highdose-rate brachytherapy in 2019. After a combination of interstitial high-dose-rate brachytherapy and nivolumab, a patient with a renal cell carcinoma metastasis showed a response and thus, reduction of tumor burden at non-irradiated sites. Nivolumab was given before and after the application of $35 \mathrm{~Gy}$ in five fractions [53]. However, the treatment response of non-irradiated lesions could also be considered a response to immunotherapy alone.

\section{Optimizing the efficacy of radioimmunotherapy}

There is a major interest in finding and developing a rational combination of radiotherapy and immunotherapy, concerning simultaneous or sequential use, dose, and fractionation as well as other variables, in order to improve synergistic effects, enhance response rates, and overcome resistances.

The appropriate timing and sequencing of radiotherapy and immunotherapy is one of the fundamental issues, i.e., understanding the dynamics and changes of the innate and adaptive immune systems in response to radiotherapy, which seems to be crucial in finding the right timing and optimal sequencing for the administration of immunotherapies. While concurrent administration of radio- and immunotherapy may have yielded better results, the concerns about cumulative toxic effects have led to a sequential administration, with the assumption of blocking checkpoints before initiating radiotherapy and turning immunologically 'cold' tumors into 'hot' tumors [54]. Substantial, conceptual, and technical improvements, including intensity-modulated radiotherapy (IMRT), image-guided radiotherapy (IGRT), stereotactic radiotherapy, and image-guided brachytherapy, have allowed precise delivery of high doses of radiotherapy, and reduce collateral damage at organs at risk and lymph nodes $[55,56]$. In this context, brachytherapy could provide a significant advantage. Due to highly conformal dose distribution, resulting in sparing of the draining lymph nodes, there is no influence on the immunespecific $T$ cells at the site of antigen presentation [57]. Regulatory $\mathrm{T}$ cells (Tregs), which create an immuno- 
Table 1. Overview of active clinical trials investigating the combination of brachytherapy and immunotherapies. The initial search was performed on clinicaltrials.gov. In total, 17 trials were identified, while only nine trials were active in October 2020

\begin{tabular}{|c|c|c|c|}
\hline Study & Phase & Entity & Interventions \\
\hline $\begin{array}{l}\text { Combination of nivolumab immunotherapy with } \\
\text { radiation therapy and androgen deprivation ther- } \\
\text { apy }\end{array}$ & I/II & Prostate cancer & $\begin{array}{l}\text { Post-ADT, participants received nivolumab, } \\
\text { HDR-BT, and EBRT, followed by a 2-year } \\
\text { follow-up period }\end{array}$ \\
\hline $\begin{array}{l}\text { IMMULAB - immunotherapy with pembrolizumab } \\
\text { in combination with local ablation in hepatocellu- } \\
\text { lar carcinoma (HCC) }\end{array}$ & II & $\begin{array}{l}\text { Hepatocellular } \\
\text { carcinoma }\end{array}$ & $\begin{array}{l}\text { Peri-interventional treatment with pem- } \\
\text { brolizumab with local ablation via either } \\
\text { RFA or MWA or BT vs. combination of TACE } \\
\text { with RFA, MWA or BT }\end{array}$ \\
\hline $\begin{array}{l}\text { Testing the addition of immunotherapy drug, } \\
\text { pembrolizumab, to the usual radiation treatment } \\
\text { of newly diagnosed early-stage high-, intermedi- } \\
\text { ate-risk endometrial cancer }\end{array}$ & III & Endometrial cancer & $\begin{array}{l}\text { Adjuvant radiotherapy }(E B R T+B T) \text { with or } \\
\text { without the addition of pembrolizumab }\end{array}$ \\
\hline $\begin{array}{l}\text { Frontline immunotherapy combined with radia- } \\
\text { tion and chemotherapy in high-risk endometrial } \\
\text { cancer (FIERCE) }\end{array}$ & I & Endometrial cancer & $\begin{array}{l}\text { Surgery followed by pembrolizumab, BT. } \\
\text { After radiotherapy, three cycles of pembroli- } \\
\text { zumab, and paclitaxel and carboplatin given }\end{array}$ \\
\hline $\begin{array}{l}\text { Pembrolizumab and chemoradiation treatment } \\
\text { for advanced cervical cancer }\end{array}$ & II & $\begin{array}{l}\text { Uterine cervical } \\
\text { cancer }\end{array}$ & $\begin{array}{l}\text { Pembrolizumab following concurrent } \\
\text { chemoradiation (EBRT + BT, cisplatin } \\
\text { weekly) }\end{array}$ \\
\hline $\begin{array}{l}\text { Brachytherapy with durvalumab or tremelimumab } \\
\text { for the treatment of patients with platinum-resis- } \\
\text { tant, refractory, recurrent, or metastatic gyneco- } \\
\text { logical malignancies }\end{array}$ & II & $\begin{array}{l}\text { Ovarian, } \\
\text { endometrial, } \\
\text { or cervical cancer }\end{array}$ & $\begin{array}{l}\text { Durvalumab vs. tremelimumab in combina- } \\
\text { tion with BT }\end{array}$ \\
\hline $\begin{array}{l}\text { PD-L1-expressing regulatory } T \text { cells in localized } \\
\text { prostate cancer patients undergoing iodine- } 125 \\
\text { permanent brachytherapy }\end{array}$ & & Prostate cancer & $\begin{array}{l}\text { Observational study to investigate changes } \\
\text { in PD-L1 expression on regulatory T cells } \\
\text { following low-dose BT }\end{array}$ \\
\hline $\begin{array}{l}\text { Immune response in prostate, lung, melanoma } \\
\text { and breast cancer patients following stereotactic } \\
\text { body radiotherapy (SBRT), intensity-modulated } \\
\text { radiotherapy (IMRT), or brachytherapy }\end{array}$ & & $\begin{array}{l}\text { Prostate, lung, } \\
\text { melanoma, } \\
\text { and breast cancer }\end{array}$ & $\begin{array}{l}\text { Change in immune biomarkers from base- } \\
\text { line and after radiation treatments }\end{array}$ \\
\hline $\begin{array}{l}\text { Immune profiling after HDR in local relapsed pros- } \\
\text { tate cancer (PRIMUS) }\end{array}$ & & Prostate cancer & $\begin{array}{l}\text { Prospective analysis of immune biomarkers } \\
\text { before and after salvage HDR-BT }\end{array}$ \\
\hline
\end{tabular}

suppressive environment and therefore, impair an effective anti-tumor immune response, are rather less sensitive to radiation than other $\mathrm{T}$ cell populations [58]. Comparing different fractionation schemes, there is an evidence that intermediate doses of about 7.50 Gy per fraction lead to a significant depletion of Tregs and thus superior tumor control compared to lower doses or very-high single radiation doses [59].

Fractionated radiotherapy can induce a robust up-regulation of checkpoint molecules. It is most likely that radiation dose and fractionation influence this effect [41]. Hypofractionated radiotherapy has distinct effects on the tumor micro-environment and tumor cell phenotype by promoting immune cell recruitment through released antigens, pro-inflammatory molecules, and danger signals as well as MHC class I expression and other immunogenic molecules, fostering the anti-tumor immune responses $[60,61]$. Immunogenic cell death and the release of danger signals is the basis of in-situ vaccination hypothesis, which is a part of the immune-stimulatory potential of radiotherapy [62]. As a result of cell death induced by ra- diotherapy, tumor antigens and debris are released into the tumor micro-environment. Next, antigen-presenting cells, like dendritic cells, are recruited and transported to lymph nodes, where the antigens are presented. However, antigen presentation and T cell interaction are subjected to circadian fluxes, and alterations, accompanied by an increased $\mathrm{T}$ cell infiltration and PD-L1 expression, create an immuno-suppressive matrix. A concurrent administration of anti-PD-1 or anti-PD-L1 agents could highjack these mechanisms and may help to achieve optimal therapeutic efficacy [63]. In this regard, an increased infiltration of innate and adaptive immune cells into solid colorectal tumors was observed after hypofractionated radiotherapy. In particular, hypofractionated radiotherapy resulted in infiltration and activation of dendritic cells (MHC-II+), but also in increased infiltration of macrophages (CD11bhigh/F4-80+) into the tumor micro-environment. Immune cell infiltration is temporal and occurs only between day 5 and 10 after the first irradiation [64]. Increased expression of MHC class I and II is associated with improved disease control in this context [65]. 
Similar results have been reported for infiltration of tumor-infiltrating lymphocytes (TILs) between day 5 and 8 after hypofractionated radiotherapy. Because lymphocytes are a radiation-sensitive cell population, shortened hypofractionated regimens with biologically equivalent doses could be beneficial. These data support performing hypofractionated radiotherapy before PD-L1 blockade [66].

In a TS/A (metastasizing mammary mouse adenocarcinoma cell line) breast cancer and MCA38 colon cancer mouse model, tumor control was demonstrated after both ablative single-dose radiotherapy and fractionated dose regimens. However, in a combination with a CTLA-A-4 antibody, immune-mediated abscopal effects were observed after hypofractionated radiotherapy. However, intermediate doses $(3 \times 8 \mathrm{~Gy})$ showed a better effect than lower doses $(5 \times 6 \mathrm{~Gy})$, indicating a specific therapeutic window for the combination of radiotherapy and CTLA-4 blockade, which may increase the efficacy of both modalities [67].

This immune-stimulatory potential, particularly when combined with immunotherapy, could improve the response rate of these two modalities. To date, optimal dose concepts and fractionation regimens remain ambiguous and seem to vary depending on the specific tumor histology. Nevertheless, a dose and fractionation regimen adjusted for an optimal local response may be expected to differ from that optimized for a distant abscopal response [68]. Therefore, intermediate doses appear to provide superior control of tumor growth as well as induction of an anti-tumor immune response. These findings provide important clues for sequential treatment planning and are relevant from the perspective of brachytherapy. Brachytherapy may play a decisive role in this approach. In particular, the doses favored in the intermediate dose range can be applied by brachytherapy and are often generated in a few fractions. During weekly intervals, immune cells have sufficient time for reconstitution. A highly conformal dose distribution allows sparing of lymphatic drainage pathways, lymph nodes, and surrounding organs at risk, which facilitates the management of side effects (Figure 2).

\section{Brachytherapy and immunotherapy - perspectives and challenges \\ Uterine cervical cancer}

Chemoradiotherapy with subsequential brachytherapy is the state-of-the-art treatment for locally advanced uterine cervical cancer. Several studies are examining the use of immuno-therapeutic drugs in the definitive treatment of uterine cervical cancer. Ongoing CALLA trial (NCT02635360), a randomized, multi-center, double-blind, placebo-controlled, global, phase III study investigates the efficacy and safety of durvalumab additional to chemoradiation or chemoradiation alone. Furthermore, KEYNOTE-A18 (NCT04221945) evaluates the efficacy and safety of pembrolizumab plus concurrent chemoradiotherapy compared to placebo and chemoradiotherapy, followed by brachytherapy in patients with locally advanced cervical cancer. These studies are amongst the first phase III trials combining a curative intended treatment, including brachytherapy, combined with immunotherapy. Brachytherapy is an integral part of the treatment regimen to achieve optimal local tumor control in adherence to dose constraints. Apart from uterine cervical cancer, other virally-driven tumors, such as head and neck squamous cell carcinoma (HNSCC), anal squamous cell carcinoma (ASCC), or Merkel cell carcino$\mathrm{ma}$, are known for their intrinsic radiation sensitivity and are considered as more immunogenic [69].

\section{Head and neck squamous cell carcinoma}

Head and neck cancers are considered more immunogenic, and immunotherapy has become an integral part of treating recurrent or metastatic disease. Chemo-/radiotherapy is the primary treatment modality for HNSCC, and in combination with immunotherapy, its potent anti-tumor response has been reported. Unfortunately, HNSCCs tempt to relapse, and after a failure of the first-line or second-line therapies, treatment options are limited due to toxicities resulting from previous treatment modalities [70]. The indication of brachytherapy for head and neck cancers differs from curative to palliative settings and from definitive to adjuvant settings, with or without surgery or EBRT, and especially as re-irradiation. Published results should encourage to further investigate brachytherapy as a part of the treatment of HNSCC to achieve local dose intensifications [71]. Therefore, concurrent application of chemotherapy can improve survival and response rates [72]. Accordingly, the combination with immunotherapy could offer further advantages. In particular, as palliative intent or salvage brachytherapy, the effectiveness of irradiation alone could increase, while toxicity remains low.

\section{Skin cancer}

Brachytherapy is a proven and valuable tool to achieve high rates of local tumor control for non-melanoma skin cancers. Especially for delicate regions, including face and scull as well as in non-resectable settings, recurrent stages after surgery, and EBRT, brachytherapy provides a treatment alternative, preserving function and good cosmetic results [73]. While immunotherapy is well-established in treating melanoma, other immuno-therapeutic approaches in treating different entities of skin cancer are evolving. In 2018, anti-PD-1 antibody cemiplimab was approved by the FDA as the first systemic treatment for advanced squamous cell skin cancer [74]. The combination of brachytherapy and PD-1-blockade may provide an evident impact on local tumor regression and long-term response, and could be the first step towards highly personalized oncology in treating non-melanoma skin cancer.

\section{Triple-negative breast cancer}

Atezolizumab has been approved by the FDA and EMA to treat non-resectable locally advanced or metastatic triple-negative, PD-L1-positive breast cancer in combination with nab-paclitaxel. Previously, atezolizumab has significantly prolonged progression-free survival compared 


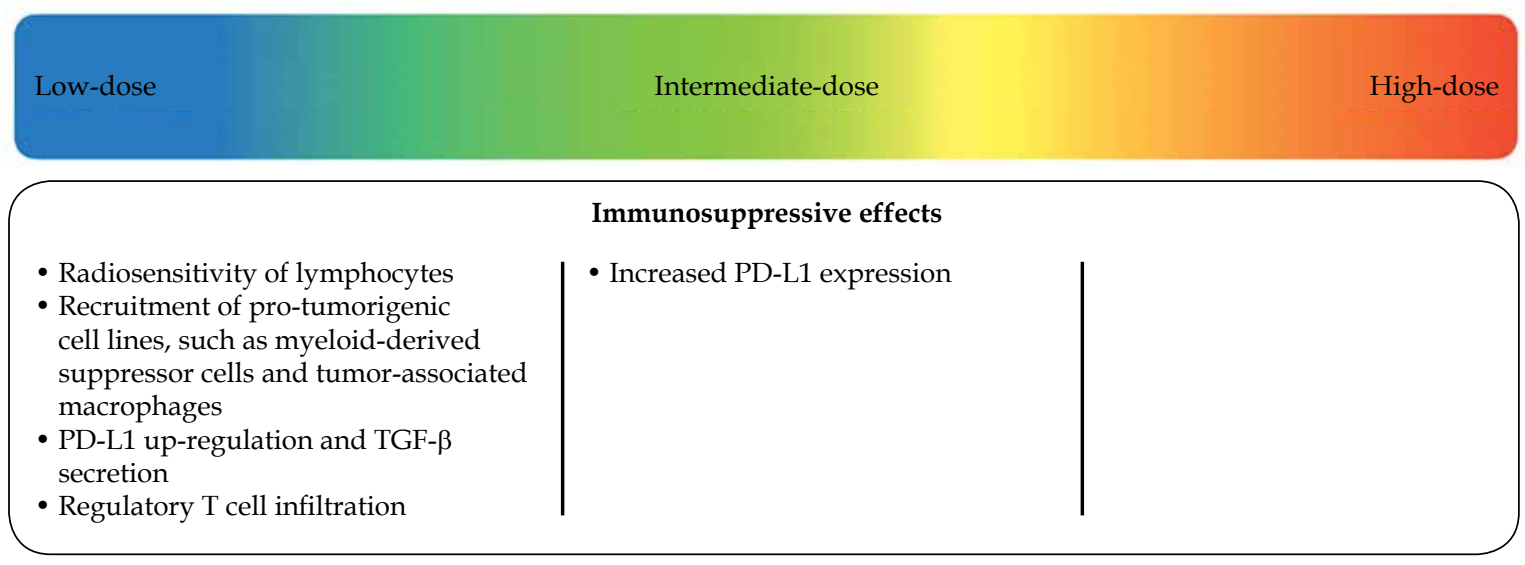

\begin{tabular}{|c|c|c|}
\hline & Immunostimulatory effects & \\
\hline $\begin{array}{l}\text { - Induction of pro-inflammatory genes } \\
\text { - MHC I and II up-regulation } \\
\text { - Activation of antigen-presenting cells. } \\
\text { Dendritic cell stimulation } \\
\text { and maturation }\end{array}$ & $\begin{array}{l}\text { - MHC I and II up-regulation } \\
\text { - Enhanced stimulation and matura- } \\
\text { tion of dendritic cells } \\
\text { - Immune cell infiltration } \\
\text { - Depletion of regulatory T cells } \\
\text { - Reconstitution of tumor vasculature }\end{array}$ & $\begin{array}{l}\text { - Radiation-induced apoptosis. } \\
\text { Release of tumor antigens } \\
\text { - IFN- } \gamma \text { induction }\end{array}$ \\
\hline
\end{tabular}

\begin{abstract}
In summary, it seems that the optimal dose per fraction to induce a robust anti-tumor immune response is somewhere between $6 \mathrm{~Gy}$ and $12 \mathrm{~Gy}$. In contrast to local tumor control, the induction of an effective immune response does not appear to grow with increasing dose, but rather to be within a specific dose-range. Here, hypofractionated radiotherapy appears to activate some of the described mechanisms of immune stimulation. Thereby, efficient abscopal responses have been reported particularly in the setting of hypofractionated radiotherapy. The combination with certain immuno-therapeutic agents seems to further enhance this effect. As a form of radiotherapy, brachytherapy may offer distinct advantages, i.e., highly conformal and sharp dose gradients allow for the induction of a broad array of immunogenic effects. In addition, by inserting the source, the region of the highest dose is delivered directly into the tumor and does not pass-through significant volumes of healthy tissue. The omission of lymphatic tissue (e.g., draining lymph nodes as a site of T cell cross priming by dendritic cells), blood vessels (avoidance of long-term lymphopenia), bone marrow, and maybe gut microbiota could, and therefore, further enhance these effects and enable good tolerability.
\end{abstract}

Fig. 2. Overview of potential immuno-suppressive and immuno-stimulatory effects of radiation depending on dose and fractionation

to patients treated with nab-paclitaxel alone [75]. Despite narrow indications, these data suggest a potential benefit of using immunotherapies in treating breast cancer. Accelerated partial breast irradiation (APBI) has been proven as an effective alternative to standard EBRT after breast conserving surgery. Following breast-conserving surgery and adjuvant radiotherapy, in addition to a lower rate of local recurrence, improved distant metastasis-free survival was also shown, which could indicate a systemic effect of local radiotherapy on micro-metastases [76]. Furthermore, brachytherapy is suitable as a re-irradiation for patients with local recurrence after breast-conserving surgery or mastectomy [77]. In this setting, systemic treatment could be beneficial or even necessary. Regarding their side-effect profile, immunotherapies may offer a tolerable synergy.

\section{Challenges}

Brachytherapy is an efficient and cost-effective treatment option for several tumor entities. However, its use decreased over the past decades among other treatment modalities due to the implementation of modern external beam irradiation techniques. Therefore, it becomes difficult to investigate the interaction between immunotherapy and brachytherapy in large prospective trials, as many centers do not offer brachytherapy. Also, the planning of such studies is challenging, especially that the optimal dose of brachytherapy or appropriate timing of immunotherapy administration relative to brachytherapy is still unknown. In spite of these challenges, it is of utmost importance to initiate prospective trials, focusing on the interaction of immunotherapy and brachytherapy, since there are clinical evidence that such treatment approaches may lead to better outcomes in several tumor entities.

\section{Conclusions}

Radiotherapy could be an optimal partner for immunotherapies. To date, immunotherapies are radically changing the management of an enormous array of different malignancies, and combinative therapies may enhance distance or systemic disease control via radia- 
tion-induced immune response and inducing abscopal effects. Nevertheless, the efficacy of immunotherapies differs widely, and there is an urgent need to define biomarkers, determine resistance mechanisms, and identify strategies to increase response rates. These could help to stratify patients, who have the best benefit in relation to adverse effects, and acute and late toxicities.

Brachytherapy, in combination with immunotherapy, appears to be a considerable partner to enhance local tumor control and may systemize responses of non-irradiated lesions. Even though we focused on the combination of checkpoint inhibitors in this article, there is a variety of approaches in immunotherapy. These therapies include adaptive $\mathrm{T}$ cell therapy, immune modulators, e.g., cytokines, therapeutic cancer vaccines, and therapies using oncolytic viruses. These therapies are going to provide a vast number of opportunities and challenges in future cancer treatment that were superficially summarized in this review. At this point, given the scarcity of relevant literature, we can only speculate about the potential of immuno-therapeutic combinations and approaches from the perspective of brachytherapy.

\section{Disclosure}

The authors report no conflict of interest.

\section{References}

1. Kruger S, Ilmer M, Kobold S et al. Advances in cancer immunotherapy 2019 - latest trends. J Exp Clin Cancer Res 2019; 38: 268.

2. Leaman Alcibar O, Candini D, López-Campos F et al. Time for radioimmunotherapy: an overview to bring improvements in clinical practice. Clin Transl Oncol 2019; 21: 992-1004.

3. Antonia SJ, Villegas A, Daniel D et al. Durvalumab after chemoradiotherapy in stage III non-small-cell lung cancer. N Engl J Med 2017; 377: 1919-1929.

4. Postow MA, Chesney J, Pavlick AC et al. Nivolumab and ipilimumab versus ipilimumab in untreated melanoma. $N$ Engl J Med 2015; 372: 2006-2017.

5. Larkin J, Chiarion-Sileni V, Gonzalez R et al. Five-year survival with combined nivolumab and ipilimumab in advanced melanoma. N Engl J Med 2019; 381: 1535-1546.

6. Ferris RL, Blumenschein G, Fayette J et al. Nivolumab for recurrent squamous-cell carcinoma of the head and neck. N Engl J Med 2016; 375: 1856-1867.

7. Bellmunt J, de Wit R, Vaughn DJ et al. Pembrolizumab as second-line therapy for advanced urothelial carcinoma. $N$ Engl J Med 2017; 376: 1015-1026.

8. Couzin-Frankel J. Breakthrough of the year 2013. Cancer immunotherapy. Science 2013; 342: 1432-1433.

9. Topalian SL, Hodi FS, Brahmer JR et al. Safety, activity, and immune correlates of anti-PD-1 antibody in cancer. N Engl J Med 2012; 366: 2443-2454.

10. Sharabi AB, Lim M, DeWeese TL et al. Radiation and checkpoint blockade immunotherapy: radiosensitisation and potential mechanisms of synergy. Lancet Oncol 2015; 16: e498-509.

11. Young KH, Baird JR, Savage T et al. Optimizing timing of immunotherapy improves control of tumors by hypofractionated radiation therapy. Mattei F (ed.). PLoS One 2016; 11: e0157164.

12. Vanpouille-Box C, Alard A, Aryankalayil MJ et al. DNA exonuclease Trex1 regulates radiotherapy-induced tumour immunogenicity. Nat Commun 2017; 8: 15618.
13. Garelli E, Rittmeyer A, Putora PM et al. Abscopal effect in lung cancer: three case reports and a concise review. Immunotherapy 2019; 11: 1445-1461.

14. Liu SZ. Nonlinear dose-response relationship in the immune system following exposure to ionizing radiation: mechanisms and implications. Nonlinearity Biol Toxicol Med 2003; 1: 71-92.

15. Tang J, Yu JX, Hubbard-Lucey VM et al. Trial watch: The clinical trial landscape for PD1/PDL1 immune checkpoint inhibitors. Nat Rev Drug Discov 2018; 17: 854-855.

16. Couzin-Frankel J. Breakthrough of the year 2013. Cancer immunotherapy. Science 2013; 342: 1432-1433.

17. Mole RH. Whole body irradiation - radiobiology or medicine? Br J Radiol 1953; 26: 234-241.

18. Demaria S, Ng B, Devitt ML et al. Ionizing radiation inhibition of distant untreated tumors (abscopal effect) is immune mediated. Int J Radiat Oncol Biol Phys 2004; 58: 862-870.

19. Kepp O, Tesniere A, Zitvogel L et al. The immunogenicity of tumor cell death. Curr Opin Oncol 2009; 21: 71-76.

20. Reits EA, Hodge JW, Herberts CA et al. Radiation modulates the peptide repertoire, enhances MHC class I expression, and induces successful antitumor immunotherapy. J Exp Med 2006; 203: 1259-1271.

21. Shaked Y. The pro-tumorigenic host response to cancer therapies. Nat Rev Cancer 2019; 19: 667-685.

22. Johansson-Percival A, He B, Ganss R. Immunomodulation of tumor vessels: it takes two to tango. Trends Immunol 2018; 39: 801-814.

23. Kozin SV, Kamoun WS, Huang Y et al. Recruitment of myeloid but not endothelial precursor cells facilitates tumor regrowth after local irradiation. Cancer Res 2010; 70: 5679-5685.

24. Kioi M, Vogel H, Schultz G et al. Inhibition of vasculogenesis, but not angiogenesis, prevents the recurrence of glioblastoma after irradiation in mice. J Clin Invest 2010; 120: 694-705.

25. Oh ET, Park MT, Song MJ et al. Radiation-induced angiogenic signaling pathway in endothelial cells obtained from normal and cancer tissue of human breast. Oncogene 2014; 33: 1229-1238.

26. Klug F, Prakash H, Huber PE et al. Low-dose irradiation programs macrophage differentiation to an iNOS ${ }^{+} / \mathrm{M} 1$ phenotype that orchestrates effective $\mathrm{T}$ cell immunotherapy. Cancer Cell 2013; 24: 589-602.

27. Paris F, Fuks Z, Kang A et al. Endothelial apoptosis as the primary lesion initiating intestinal radiation damage in mice. Science 2001; 293: 293-297.

28. Tong F, Xiong CJ, Wei CH et al. Hypo-fractionation radiotherapy normalizes tumor vasculature in non-small cell lung cancer xenografts through the p-STAT3/HIF-1 alpha signaling pathway. Ther Adv Med Oncol 2020; 12: 1758835920965853.

29. Kono H, Rock KL. How dying cells alert the immune system to danger. Nat Rev Immunol 2008; 8: 279-289.

30. Nakamura N, Kusunoki Y, Akiyama M. Radiosensitivity of CD4 or CD8 positive human T-lymphocytes by an in vitro colony formation assay. Radiat Res 1990; 123: 224-227.

31. Drake CG. Radiation-induced immune modulation. In: DeWeese TL, Laiho M. Molecular determinants of radiation response. Springer New York, New York, NY 2011; 251-263.

32. Walle T, Martinez Monge R, Cerwenka A et al. Radiation effects on antitumor immune responses: current perspectives and challenges. Ther Adv Med Oncol 2018; 10: 1758834017742575.

33. Wennerberg E, Lhuillier C, Vanpouille-Box C et al. Barriers to radiation-induced in situ tumor vaccination. Front Immunol 2017; 8: 229.

34. Patel RB, Baniel CC, Sriramaneni RN et al. Combining brachytherapy and immunotherapy to achieve in situ tumor vaccination: A review of cooperative mechanisms and clinical opportunities. Brachytherapy 2018; 17: 995-1003. 
35. Herrera FG, Bourhis J, Coukos G. Radiotherapy combination opportunities leveraging immunity for the next oncology practice. CA Cancer J Clin 2017; 67: 65-85.

36. Postow MA, Callahan MK, Barker CA et al. Immunologic correlates of the abscopal effect in a patient with melanoma. N Engl J Med 2012; 366: 925-931.

37. Theurich S, Rothschild SI, Hoffmann M et al. Local tumor treatment in combination with systemic ipilimumab immunotherapy prolongs overall survival in patients with advanced malignant melanoma. Cancer Immunol Res 2016; 4 744-754.

38. Golden EB, Chhabra A, Chachoua A et al. Local radiotherapy and granulocyte-macrophage colony-stimulating factor to generate abscopal responses in patients with metastatic solid tumours: a proof-of-principle trial. Lancet Oncol 2015; 16: 795-803.

39. Morris ZS, Guy EI, Francis DM et al. In situ tumor vaccination by combining local radiation and tumor-specific antibody or immunocytokine treatments. Cancer Res 2016; 76: 3929-3941.

40. Herskind C, Fleckenstein K, Lohr J et al. Antitumoral action of interferons and interleukins in combination with radiotherapy. Part II: radiobiological and immunologic strategies. Strahlenther Onkol 2004; 180: 331-339.

41. Dovedi SJ, Adlard AL, Lipowska-Bhalla G et al. Acquired resistance to fractionated radiotherapy can be overcome by concurrent PD-L1 blockade. Cancer Res 2014; 74: 5458-5468.

42. Chen Y, Gao M, Huang Z et al. SBRT combined with PD-1/PDL1 inhibitors in NSCLC treatment: a focus on the mechanisms, advances, and future challenges. J Hematol Oncol 2020; 13: 105.

43. Shaverdian N, Lisberg AE, Bornazyan K et al. Previous radiotherapy and the clinical activity and toxicity of pembrolizumab in the treatment of non-small-cell lung cancer: a secondary analysis of the KEYNOTE-001 phase 1 trial. Lancet Oncol 2017; 18: 895-903.

44. Hwang WL, Niemierko A, Hwang KL et al. Clinical outcomes in patients with metastatic lung cancer treated with PD-1/PD-L1 inhibitors and thoracic radiotherapy. JAMA Oncol 2018; 4: 253

45. Theelen WSME, Peulen HMU, Lalezari F et al. Effect of pembrolizumab after stereotactic body radiotherapy vs pembrolizumab alone on tumor response in patients with advanced non-small cell lung cancer: results of the PEMBRO-RT phase 2 randomized clinical trial. JAMA Oncol 2019; 5: 1276-1282.

46. Deutsch E, Chargari C, Galluzzi L et al. Optimising efficacy and reducing toxicity of anticancer radioimmunotherapy. Lancet Oncol 2019; 20: e452-463.

47. Sha CM, Lehrer EJ, Hwang C et al. Toxicity in combination immune checkpoint inhibitor and radiation therapy: A systematic review and meta-analysis. Radiother Oncol 2020; 151: 141-148.

48. Twyman-Saint Victor C, Rech AJ, Maity A et al. Radiation and dual checkpoint blockade activate non-redundant immune mechanisms in cancer. Nature 2015; 520: 373-377.

49. Hodge JW, Sharp HJ, Gameiro SR. Abscopal regression of antigen disparate tumors by antigen cascade after systemic tumor vaccination in combination with local tumor radiation. Cancer Biother Radiopharm 2012; 27: 12-22.

50. Rodriguez-Ruiz ME, Rodriguez I, Garasa $S$ et al. Abscopal effects of radiotherapy are enhanced by combined immunostimulatory mAbs and are dependent on CD8 T cells and crosspriming. Cancer Res 2016; 76: 5994-6005.

51. Berenguer Frances MA, Linares-Galiana I, Cañas Cortés R et al. Changes of CD68, CD163, and PD-L1 tumor expression during high-dose-rate and pulsed-dose-rate brachytherapy for cervical cancer. Brachytherapy 2020; 19: 51-59.

52. Bellia SR, Feliciani G, Duca MD et al. Clinical evidence of abscopal effect in cutaneous squamous cell carcinoma treated with diffusing alpha emitters radiation therapy: a case report. J Contemp Brachytherapy 2019; 11: 449-457.

53. Suzuki G, Masui K, Yamazaki H et al. Abscopal effect of high-dose-rate brachytherapy on pelvic bone metastases from renal cell carcinoma: a case report. J Contemp Brachytherapy 2019; 11: 458-461.

54. Hader M, Frey B, Fietkau R et al. Immune biological rationales for the design of combined radio- and immunotherapies. Cancer Immunol Immunother 2020; 69: 293-306.

55. Jaffray DA. Image-guided radiotherapy: from current concept to future perspectives. Nat Rev Clin Oncol 2012; 9: 688-699.

56. Strnad V, Ott OJ, Hildebrandt G et al. 5-year results of accelerated partial breast irradiation using sole interstitial multicatheter brachytherapy versus whole-breast irradiation with boost after breast-conserving surgery for low-risk invasive and in-situ carcinoma of the female breast: a randomised, phase 3, non-inferiority trial. Lancet 2016; 387: 229-238.

57. Wang $\mathrm{Y}$, Deng $\mathrm{W}, \mathrm{Li} \mathrm{N}$ et al. Combining immunotherapy and radiotherapy for cancer treatment: current challenges and future directions. Front Pharmacol 2018; 9: 185.

58. Multhoff G, Gaipl US, Niedermann G. The role of radiotherapy in the induction of antitumor immune responses. Strahlenther Onkol 2012; 188 Suppl 3: 312-315.

59. Schaue D, Ratikan JA, Iwamoto KS et al. Maximizing tumor immunity with fractionated radiation. Int J Radiat Oncol Biol Phys 2012; 83: 1306-1310.

60. Reits EA, Hodge JW, Herberts CA et al. Radiation modulates the peptide repertoire, enhances MHC class I expression, and induces successful antitumor immunotherapy. J Exp Med 2006; 203: 1259-1271.

61. Zhang B, Bowerman NA, Salama JK et al. Induced sensitization of tumor stroma leads to eradication of established cancer by T cells. J Exp Med 2007; 204: 49-55.

62. Morris ZS, Guy EI, Francis DM et al. In situ tumor vaccination by combining local radiation and tumor-specific antibody or immunocytokine treatments. Cancer Res 2016; 76: 3929-3941.

63. Dovedi SJ, Illidge TM. The antitumor immune response generated by fractionated radiation therapy may be limited by tumor cell adaptive resistance and can be circumvented by PD-L1 blockade. Oncoimmunology 2015; 4: e1016709.

64. Frey B, Rückert M, Weber J et al. Hypofractionated irradiation has immune stimulatory potential and induces a timely restricted infiltration of immune cells in colon cancer tumors. Front Immunol 2017; 8: 231

65. Galluzzi L, Yamazaki T, Kroemer G. Linking cellular stress responses to systemic homeostasis. Nat Rev Mol Cell Biol 2018; 19: 731-745.

66. Zhang X, Niedermann G. Abscopal effects with hypofractionated schedules extending into the effector phase of the tumor-specific T-cell response. Int J Radiat Oncol Biol Phys 2018; 101: 63-73.

67. Dewan MZ, Galloway AE, Kawashima N et al. Fractionated but not single-dose radiotherapy induces an immune-mediated abscopal effect when combined with anti-CTLA-4 antibody. Clin Cancer Res 2009; 15: 5379-5388.

68. Buchwald ZS, Wynne J, Nasti TH et al. Radiation, immune checkpoint blockade and the abscopal effect: a critical review on timing, dose and fractionation. Front Oncol 2018; 8: 612.

69. Rödel F, Martin D, Balermpas P et al. Modulation of radiation sensitivity and antitumor immunity by viral pathogenic factors: implications for radio-immunotherapy. Biochim Biophys Acta Rev Cancer 2019; 1871: 126-137.

70. Karam SD, Raben D. Radioimmunotherapy for the treatment of head and neck cancer. Lancet Oncol 2019; 20: e404-416.

71. Meyer JE, Brocks C, Maune S et al. Brachytherapy for the treatment of head and neck cancer. HNO 2010; 58: 947-958. 
72. Ritter M, Teudt IU, Meyer JE et al. Second-line treatment of recurrent HNSCC: tumor debulking in combination with high-dose-rate brachytherapy and a simultaneous cetuximab-paclitaxel protocol. Radiat Oncol 2016; 11: 6.

73. Skowronek J. Brachytherapy in the treatment of skin cancer: an overview. Postepy Dermatol Alergol 2015; 5: 362-367.

74. Migden MR, Rischin D, Schmults CD et al. PD-1 blockade with cemiplimab in advanced cutaneous squamous-cell carcinoma. N Engl J Med 2018; 379: 341-351.

75. Schmid P, Adams S, Rugo HS et al. Atezolizumab and nab-paclitaxel in advanced triple-negative breast cancer. N Engl J Med 2018; 379: 2108-2121.

76. Overgaard M, Hansen PS, Overgaard J et al. Postoperative radiotherapy in high-risk premenopausal women with breast cancer who receive adjuvant chemotherapy. Danish Breast Cancer Cooperative Group 82b Trial. N Engl J Med 1997; 337: 949-955.

77. Skowronek J, Chicheł A. Brachytherapy in breast cancer: an effective alternative. Prz Menopauzalny 2014; 13: 48-55. 